\title{
High rate of ceftaroline-resistance in Staphylococcus haemolyticus causing late-onset sepsis and colonizing preterm neonates and their mothers
}

\section{Hiie Soeorg', Evamaria Elisabet Keränen', Hanna Kadri Metsvaht', Sirli Treumuth¹, Imbi Eelmäe², Mirjam Merila ${ }^{3}$,}

Mari-Liis IImoja ${ }^{4}$, Tuuli Metsvaht ${ }^{2}$, Irja Lutsar ${ }^{1}$

${ }^{1}$ Department of Microbiology, University of Tartu, Tartu, Estonia. ${ }^{2}$ Pediatric Intensive Care Unit, Tartu University Hospital, Tartu, Estonia

${ }^{3}$ Department of Neonatology, Children's Clinic, Tartu University Hospital, Tartu, Estonia. ${ }^{4}$ Pediatric Intensive Care Unit, Tallinn Children's Hospital, Tallinn, Estonia

\section{Background \& Aim}

- Ceftaroline is a new cephalosporin active against MRSA

- Susceptibility of $S$. epidermidis and $S$. haemolyticus causing late-onset sepsis (LOS) in preterm neonates warrants characterization before use of ceftaroline in treatment of LOS

- We aimed to determine susceptibility to ceftaroline in $m e c A$ positive S. epidermidis and S. haemolyticus causing LOS and colonizing potential reservoirs of invasive strains (gut/skin of preterm neonates, mother's own milk (MOM))

\section{Materials and Methods}

- A total of 97 previously characterized mecA-positive strains of diverse multilocus sequence types (Table1)

\begin{tabular}{llccc}
\multicolumn{4}{c}{ Table 1. Number of isolates included in the study according to time, source and species } \\
\hline & $\begin{array}{c}\text { Colonizing } \\
\text { Gut/skin }(\mathbf{n}=\mathbf{4 1})\end{array}$ & $\begin{array}{c}\text { Causing } \\
\text { MOM=39) }\end{array}$ & LOS ( $\mathbf{n = 1 7 )}$ \\
\hline $2007-$ & S. epidermidis & 11 & 0 & 2 \\
2008 & S. haemolyticus & 0 & 0 & 4 \\
$2014-$ & S. epidermidis & 3 & 31 & 3 \\
2015 & S. haemolyticus & 27 & 8 & 8 \\
\hline
\end{tabular}

- Oxacillin and ceftaroline MICs were determined by MIC Test Strips (Liofilchem)

- Results interpreted according to EUCAST 2018 (Table 2) Table 2.MIC breakpoints for determination of resistance (according to EUCAST 2018)

\begin{tabular}{lccc}
\hline & Susceptible & Intermediate & Resistant \\
Ceftaroline* $^{\leq}$ & $\leq 1 \mathrm{mg} / \mathrm{L}$ & $1<\ldots \leq 2 \mathrm{mg} / \mathrm{L}$ & $>2 \mathrm{mg} / \mathrm{L}$ \\
Oxacillin & $\leq 0.25 \mathrm{mg} / \mathrm{L}$ & - & $>0.25 \mathrm{mg} / \mathrm{L}$ \\
\hline
\end{tabular}

${ }^{*}$ Ceftaroline breakpoint for $S$. aureus (indications other than pneumonia).

\section{Results}

- S. epidermidis compared with $S$. haemolyticus had lower ceftaroline MICs $(p<0.001)$ and lower resistance-rate to ceftaroline $(p<0.001)$ (Table 3$)$

\begin{tabular}{llcccccc}
\multicolumn{7}{l}{ Table 3. The results of susceptibility testing to oxacillin and ceftaroline } \\
\hline Oxacillin & MIC $_{50}$ & MIC $_{90}$ & Susceptible (\%) & Intermediate (\%) & Resistant (\%) \\
& S. epidermidis & 2 & 16 & 4 & 0 & 96 \\
Ceftaroline & S. haemolyticus & $\geq 256$ & $\geq 256$ & 0 & 0 & 100 \\
& S. epidermidis & 0.25 & 0.5 & 98 & 0 & 2 \\
& S. haemolyticus & 3 & 4 & 6.4 & 23.4 & 70.2 \\
\hline
\end{tabular}

- $S$. epidermidis from MOM had lower ceftaroline MICs compared with $S$. epidermidis colonizing gut/skin of neonates or causing LOS $\left(\mathrm{MIC}_{50} / \mathrm{MIC}_{90} 0.25 / 0.5\right.$ vs $0.38 / 0.5 \mathrm{mg} / \mathrm{L} ; \mathrm{p}=0.012$ and $\mathrm{p}=0.02$, respectively), but colonizing and LOS-causing S. haemolyticus had similar MICs (Figure 1)

- Ceftaroline-resistant $S$. haemolyticus isolates belonged to various sequence types (ST1, 3, 25, 42) and were detected in both time periods (2007-2008 and 2014-2015)

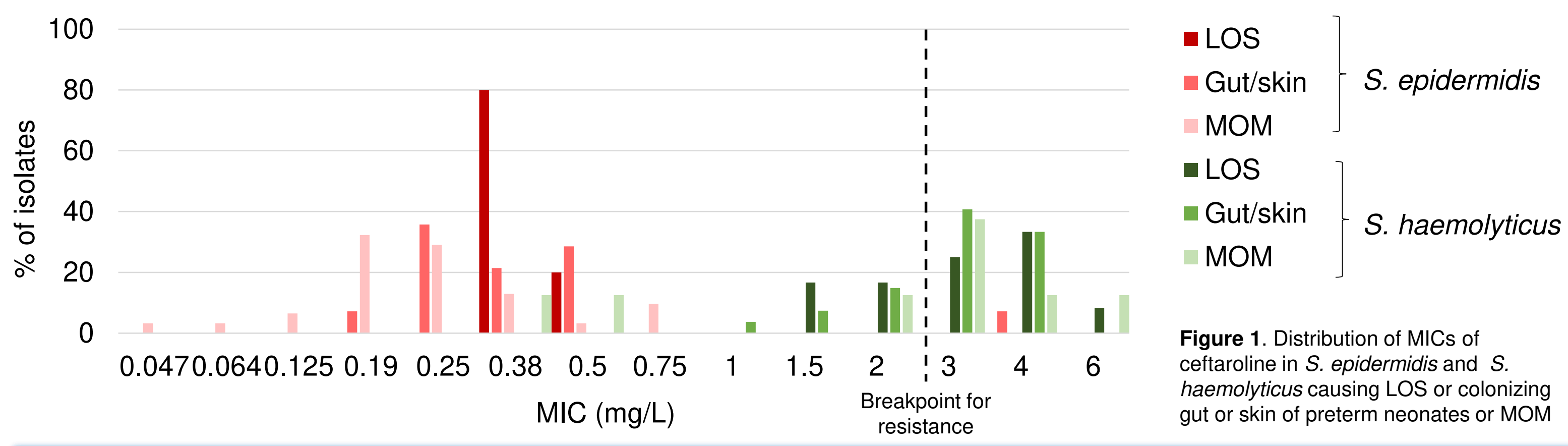

\section{Conclusion}

- S. epidermidis colonizing gut/skin and MOM or causing LOS in preterm neonates is mostly susceptible to ceftaroline.

- Widespread ceftaroline-resistance in S. haemolyticus may limit its potential use in treatment of LOS in preterm neonates. 